\title{
Correspondence
}

\section{Rewarming infants on a heated mattress}

Sir,

Sarman et al have shown that a heated, water filled mattress can provide a superior alternative to maternal skin contact, or an incubator, for rapidly attaining and maintaining normal body temperature in the low birthweight neonate. ${ }^{\prime}$ In this country, where power failures are very infrequent, an electric mattress heater may be as useful and simple a means of ensuring rapid restoration of normal temperature in the newborn.

To test the effect of such a heater I measured the rectal temperatures of 38 full term infants delivered by uncomplicated vaginal delivery in cephalic presentation after labours lasting no longer than 10 hours. Two groups of infants were studied: 20 born of mothers who had received $100-150 \mathrm{mg}$ pethidine during the last six hours of labour (mean birth weight $3315 \mathrm{~g}$ ), and 18 who had not (mean birth weight $3339 \mathrm{~g}$ ). Half of these two groups of infants was nursed in a cot with a 40 watt electric heating pad (Thermega) beneath the mattress, and half was not.

All infants were dried and wrapped in two towels immediately after delivery before transfer to the postnatal ward where their rectal temperatures were recorded with a mercury thermometer. No toilet was performed but a rectal thermocouple (Light Laboratories) was inserted to 3 $\mathrm{cm}$ before infants were rewrapped and left undisturbed for the next four hours in a cot in a side room with an ambient temperature of $26^{\circ} \mathrm{C}$. Rectal temperatures were recorded at $1,2,3$, and 4 hours of age. At 4 hours the rectal thermocouple was removed and the infants received their first toilet. Thereafter, until 24 hours, when the rectal temperature was recorded with a mercury thermometer, all infants were nursed without the heating pad.

The rate of rise of the mean rectal temperature of those infants nursed in cots with a heating pad beneath their mattress was faster than that of infants nursed without, whether or not their mothers had received pethidine $(\mathrm{p}<0.01)$ (figure). At four and 24 hours there was no significant difference between the mean temperatures of the four groups of infants. The temperature recorded between the upper surface of the mattress and the wrapped infant in a cot containing a heater was $38.4^{\circ} \mathrm{C}$ and that without a heater $34 \cdot 8^{\circ} \mathrm{C}$.

This small study shows that an electric undermattress heating pad rapidly restores body temperature to normal in the healthy neonate, whether or not he or she has been exposed to pethidine in utero. Such a heating pad is safe and effective and may obviate the use of a radiant heater to diminish loss of body heat from non-asphyxiated infants born of mothers who have received pethidine in labour..$^{2+}$ After prompt drying at birth infants may be safely left wrapped and undisturbed until first toilet at 4 hours of age.

Such an electric undermattress heater may well prove

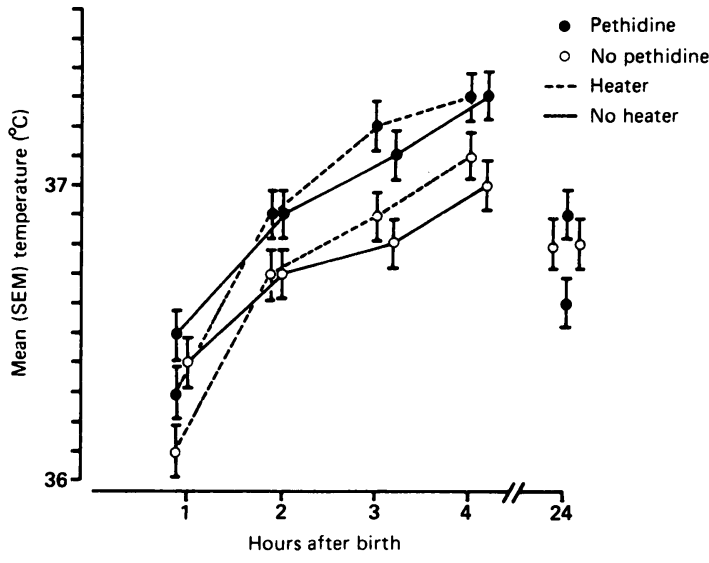

Figure Rectal temperatures of neonates nursed in a cot with and without a heated mattress.

equally effective in the management of the low birthweighte newborn who does not require nursing in an incubator. $\rightleftharpoons$

I should like to thank Dr EN Hey and the midwives of the Princess Mary Maternity Hospital for their help with this study.

\section{References}

'Sarman I, Can G. Tuncll R. Rewarming preterm infants on a heated, water filled mattress. Arch Dis Child 1989:64:687-92.

2 Burnard ED. Cross KW. Rectal temperature in the newborn infant after birth asphyxia. Br Med J 1958;ii:1197-9.

${ }^{3}$ Dahm LS. James LS. Newborn temperature and calculated heat loss in the delivery room. Pediatrics 1972:49:504-13.

+ Smales ORC, Kime R. Thermoregulation in babies immediately after birth. Arch Dis Child 1978:53:58-61.

L T WEAVER MRC Dunn Nutrition Unit, Milton Road, Cambridge CB4 IXJ

\section{Cholesterol and diet}

\section{Sir,}

Dr Tarlow reports that the fat intake of many British children currently exceeds $50 \%$ of the total energy of the diet. 
The target he sets is $35 \%$ of total energy without prejudicing growth or producing an unpleasant diet. Your readers might be interested in some preliminary findings of dietary intake at 18 months in a sample of 170 Hong Kong Chinese infants who are participating in a longitudinal study of growth and nutritional inter-relationships from birth to 5 years.

Fat contributed about $30 \%$ of total daily energy consumption at 18 months. Most infants were then eating a diet of rice, fish, meat, and vegetables. Butter and high fat desserts were rarely given and ice cream only occasionally consumed. Energy intake was $0.414 \mathrm{MJ} / \mathrm{kg} /$ day, very similar to energy intake in recent studies from Canadian² $(0.401 \mathrm{MJ})$ and Australian ${ }^{3}$ children $(0.418 \mathrm{MJ})$ of the same age. Our infants enjoyed excellent health and were growing and developing normally.

In Hong Kong even with its obvious western influences the traditional Chinese diet in early childhood is low in total fat and cholesterol and saturated fats. As children grow older, however, the situation is likely to change as ubiquitous 'fast foods' increase their stranglehold on children's diets. It remains to be seen whether the very low fat intake of the early years in any way influences health in the long term especially in relation to cardiovascular diseases.

\section{References}

1 Tarlow MJ. Cholesterol and diet. Arch Dis Child 1989;64: 647-8.

2 Yeung DL. Infant nutrition. A study of feeding practices and growth from birth to 18 months. Ottawa, Canada: The Canadian Public Health Association. 1983.

${ }^{3}$ Hitchcock NE, Gracey M. Gilmour AI. Owles EN. Nutrition and growth in infancy and carly childhood. A longitudinal study from birth to five years. Monographs in paediatrics 19. Basel: Karger, 1986.

S S F Leung, S LuI, and D P Davies Department of Paediatrics, The Prince of Wales Hospital, (The Chinese University of Hong Kong), Shatin, Hong Kong

\section{Educating medical students about death and dying}

Sir,

I read with interest the article by Black et al, which points out the need to address the stressful feelings medical students and physicians have about dying and death. ${ }^{\prime}$ The authors propose a course with objectives varying from identifying the dying patients ${ }^{*}$ and family needs and their dealing with grief, to clearing away barriers set up by physicians surrounding death. A course of this type would no doubt improve a physician's ability to deal with death and dying, and would be helpful to their dying patients and their families. However. I feel the course proposed lacks one more important objective: obtaining permission for a necropsy and feedback to the family about the necropsy findings.

The information gained from a necropsy, and properly presented to the family, may relieve the guilt that is a consequence of most deaths, provides assurance that the patient received proper medical care, and facilitates the grieving process. ${ }^{2-4}$ As Hill and Anderson point out, the necropsy not only gives the physician the benefit of learning from his or her own experience, but also enables the medical student to accept the fact of death as an inescapable part of caring for the sick. ${ }^{4}$

\section{References}

${ }^{1}$ Black D, Hardoff D, Nelki J. Educating medical students about death and dying. Arch Dis Child 1989;64:750-3.

2 Valdes-Dapena MA. The pathologist's conference with patients following post mortem examination of their child: an application of the Kubler-Ross concept. Perspect Pediatric Pathol 1979:5:263-7.

${ }^{3}$ Favara BE, Cotteau C, McIntyre L, Valdes-Dapena M. Pediatric pathology and the autopsy. Pediatr Pathol 1989;9:19-116.

+ Hill RB, Anderson RE. The autopsy-medical practice and public policy. Massachusetts: Butterworth, 1988:127.

W A DEvine Pathology Department, Children's Hospital of Pittsburgh, One Children's Place, 3705 Fifth Avenue at De Soto Street, Pittsburgh, Pennsylvania, 15213-2583, USA

\section{Rise in urea concentration after arginine hydrochloride infusion}

Sir,

In order to test the renal ability of children to acidify their urine, an adequate degree of metabolic acidosis is usually obtained either by the oral administration of ammonium chloride or by the infusion of arginine hydrochloride. ${ }^{1}$ The intravenous infusion of arginine hydrochloride-which is used also to study growth hormone, glucagon, and insulin release $^{2.3}$ - has been described as safe and free of immediate or delayed untoward effects. Transient renal dysfunction, hypophosphataemia, and hyperkalaemia have, however, been described after arginine administration to normal subjects. ${ }^{4}$

When reviewing arginine acidification tests performed in our clinic, we observed a significant progressive increase in plasma urea concentrations in children with normal glomerular filtration rate undergoing the test. In seven children aged 5 months to 6 years, the intravenous administration of arginine hydrochloride $\left(100-150 \mathrm{mmol} / \mathrm{m}^{2}\right.$ over 120-150 minutes) increased mean (SD) plasma urea from $5 \cdot 1(0 \cdot 5)$ to $7 \cdot 3(0 \cdot 6) \mathrm{mmol} / \mathrm{l}$ at 60 minutes $(\mathrm{p}<0 \cdot 001)$ 